\section{Detection of CD4+ and CD8+ Lymphocytes in the Intestine of Broiler Chicks Treated with Lactobacillus spp. and Challenged with Salmonella enterica serovar Enteritidis}

author(s)

Noujaim JC

Andreatti Filho RL

Lima ET de

Okamoto AS

Department of Clinical Veterinary Medicine College of Veterinary Medicine and Animal Science - FMVZ

São Paulo State University - UNESP

Botucatu, SP, Brazil.

\section{Mail Address}

\section{Raphael Lucio Andreatti Filho}

Department of Clinical Veterinary Medicine

College of Veterinary Medicine and Animal

Science. São Paulo State University

Distrito Rubião Júnior $\mathrm{S} / \mathrm{N}$

18.618-000, Botucatu, SP, Brazil

Telephone/fax: +55 (14) 38116293

E-mail: andreatti@fmvz.unesp.br

\section{Keywords}

Chicken, immunohistochemistry, lymphocytes CD4+ and CD8+, probiotic, Salmonella.

\section{ABSTRACT}

The expression of immune response as a leukocytic infiltrate by CD4+ and CD8+ cells in the epithelium and in the intestinal lamina propria of chicks fed Lactobacillus spp or cecal microflora (CM) and experimentally challenged or not with Salmonella enterica serovar Enteritidis (SE) was studied using immunohistochemistry. Three hundred and twenty dayof-hatch broiler chicks were divided into four groups of 80 birds each and orally received L. reuteri, L. salivarius, L. acidophilus, or CM. Each group was subdivided into four subgroups of 20 birds each, classified as follows: a subgroup did not receive any oral treatment (negative control), subgroup treated with $L$. spp or CM, subgroup treated with $L$. spp or $C M$ and challenged with $S E$, and subgroup only challenged with SE (positive control). The results show that the oral treatment with $L$. reuteri, L. salivarius, L. acidophilus, or CM and challenge or not with SE stimulated bird immune response as determined by the leukocytic infiltrate by CD8+ lymphocytes followed by CD4+ in the epithelium and in the lamina propria of the duodenum, jejunum, and cecum of chicks up to 12 days of age. CD8+ lymphocyte number was significantly higher in the intestine of chicks receiving CM and challenged with SE. The duodenum, followed by the jejunum, were the segments in which the immune response, as shown by T, CD4+ and CD8+ cells, was stimulated with the greatest intensity.

\section{INTRODUCTION}

Major risk factors for food poisoning caused by Salmonella are associated with chickens, such as eating raw or undercooked eggs (Molbak \& Neiman, 2002). Salmonella enterica serovar Enteritidis (SE) is the second most common serotype in humans and most commonly identified from clinical and non-clinical chicken sources (Centers for Disease Control and Prevention, 2005). This serovar can infect chickens in the absence of clinical disease (Gast \& Beard, 1990). Several measures to control Salmonella have been used, such as antimicrobial drugs. However, concerns with drug-resistant bacteria and the detection of drug residues in food of animal origin have stimulated the interest in alternative treatments, such as the use of probiotics, competitive exclusion products, and bacteriophages (Sulakvelidze et al., 2001; Vanlmmerseel et al., 2002; Andreatti Filho et al., 2003; Joerger, 2003).

Lilly \& Stillwell (1965) were the first to utilize the term probiotic and the work of Nurmi \& Rantala (1973) was the initial milestone of studies involving the concept of competitive exclusion. The principle of competitive exclusion is particularly interesting because it provides some protection of birds against their contamination by pathogens in the first days after hatch, although at this age, neither the intestinal microflora or the immune system of birds is fully developed (Day, 1992). 
Noujaim JC, Andreatti Filho RL, Lima ET de, Okamoto AS
Detection of CD4+ and CD8+ Lymphocytes in the Intestine of Broiler Chicks Treated with Lactobacillus spp. and Challenged with Salmonella enterica serovar Enteritidis
The intestinal microflora is composed of various bacterial species, including the prominent genus Lactobacillus, which most common species, such as $L$. reuteri, L. salivarius, L. animalis, and L. acidophillus, have shown to be important for the intestinal health of chicks (Ramesh et al., 2000).

Oral treatments with Lactobacillus spp. have demonstrated immunostimulant effects on the intestinal mucosa (Simon et al., 2001). In chickens, the presence of Lactobacillus spp. in the intestine stimulates synthesis of IgA by the immune system through the release of short chain peptides, increasing the resistance to diseases (Pulverer et al., 1990). Furthermore, lactobacilli are also associated to beneficial effects in the therapy against tumors in humans, exerting antimutagenic effects and modulating immune response, including T-cells, probably due to the influence of cytokines on CD4+ cells (Schifrin et al., 1997; Pelto et al., 1998). Therefore, the presence of Lactobacillus is essential for regulating the composition of intestinal microflora, developing intestinal immunity, and promoting chicken health (Muir et al., 2000).

The objective of this study was to evaluate immune system stimulation, in the form of CD4+ and CD8+ lymphocyte leukocytic infiltrate in the intestinal epithelium and lamina propria, of broiler chicks orally treated with Lactobacillus reuteri, L. salivarius, $L$. acidophilus, or cecal microflora (CM), isolated from breeders, and challenged or not with Salmonella enterica serovar Enteritidis (SE).

\section{MATERIAL AND METHODS \\ Birds}

Three hundred and twenty day-of-hatch broiler chicks were obtained from a local hatchery. Chicks were housed in metal cages, maintained under heating for 12 days, and were offered water and a commercial feed ad libitum, with no addition of antibiotics. Only Salmonella-free chicks were utilized, as certified by the results of Salmonella isolation and identification tests of a flock sample, according to the methodology described by Nagajara et al. (1991).

\section{Experimental design}

The 320 birds utilized were divided into four groups containing 80 chicks each and submitted to oral treatments with $L$. reuteri, $L$. salivarius, $L$. acidophilus, and CM. Each group was subdivided into four subgroups of 20 birds each, which were divided as follows: a subgroup which was not orally treated or challenged (negative control), subgroup orally treated with L. spp or CM, subgroup orally treated with $\mathrm{L}$. spp or CM and challenged with $\mathrm{SE}$, and a subgroup only challenged with SE (positive control). At two, four, eight, and 12 days of age, five birds from each subgroup were euthanized by cervical dislocation, and fragments of duodenum, jejunum and ceca were aseptically removed and fixed in 10\% Bouin solution for 24 hours.

\section{Preparation of cecal microflora and Lactobacillus cultures}

CM was obtained from three 35-week-old breeders, euthanized by cervical dislocation, and aseptically necropsied for the removal of the ceca, which contents were cultivated in $10 \mathrm{~mL}$ thioglycolate broth in an anaerobic jar containing the Anaerobac ${ }^{\circledR}$ system for 24 hours at $40^{\circ} \mathrm{C}$. Concomitantly, this material also was tested to verify the presence of Salmonella spp. (Nagajara et al., 1991). Samples positive for Salmonella spp. were discarded. After incubation, the number of colony-forming units (CFU) of intestinal bacteria was determined, after which decimal dilution series were performed in $\mathrm{pH} 7.2$ phosphate buffer saline solution (PBS). From those decimal dilutions, $0.1 \mathrm{~mL}$ was inoculated in Petri dishes containing thioglycolate agar and cultivated under anaerobiosis for 24 hours at $40^{\circ} \mathrm{C}$. Day-of-hatch chicks received 107 CFU/chick $(0.5 \mathrm{~mL})$ of CM, Lactobacillus acidophilus, $L$. reuteri, or $L$. salivarius by oral gavage utilizing graduated pipette. All the lactobacilli were isolated from the ceca of 35week-old breeders, biochemically identified, stored in nutrient broth containing 10\% glycerol, and frozen in liquid nitrogen.

Biochemical identification was performed by carbohydrate fermentation test. Lactobacilli samples were propagated in 3mL DeMan-Rugosa-Sharpe broth (MRS) free from meat extract and glucose, with $0.002 \%$ bromocresol purple as indicator, and $0.01 \%$ of the following carbohydrates were individually added: arabinose, fructose, galactose, glucose, mannitol, mannose, maltose, sucrose, salicyl, and sorbitol. Tubes were incubated at $37^{\circ} \mathrm{C}$ for 48 hours (Kandler \& Weiss, 1986).

Each cryotube containing $1.5 \mathrm{~mL}$ of culture of Lactobacillus spp. was thawed, resuspended in MRS broth, and incubated under anaerobiosis for 24 hours at $40^{\circ} \mathrm{C}$ before determination of inoculum CFU, which was carried out by decimal dilution series of MRS broth in PBS and plating $0.1 \mathrm{~mL}$ of these dilutions on Petri dishes containing MRS agar. Cultured were incubated under anaerobiosis for 48 hours at $40^{\circ} \mathrm{C}$. 
Noujaim JC, Andreatti Filho RL, Lima ET de, Okamoto AS
Detection of CD4+ and CD8+ Lymphocytes in the Intestine of Broiler Chicks Treated with Lactobacillus spp. and Challenged with Salmonella enterica serovar Enteritidis

\section{Preparation of Salmonella Enteritidis inoculum}

A SE strain resistant to nalidixic acid and to rifampicin, and isolated from the liver of chickens was utilized for the challenge. This resistance was developed through successive culture in brilliant green agar (BGA), containing $100 \mu \mathrm{g} / \mathrm{mL}$ nalidixic acid and rifampicin (Weinack et al., 1982). The inoculum was obtained by culture in brain-heart infusion broth (BHI) incubated at $40^{\circ} \mathrm{C}$ for 12 hours. The challenge inoculum was quantified by CFU number per chick, as previously described. In order to determine CFU number, 100 $\mathrm{\mu g} /$ $\mathrm{mL}$ of these dilutions were plated in BGA increased from antibiotics. After a period of incubation of 24 hours at $40^{\circ} \mathrm{C}$, reading was performed for CFU determination. On the third day of life, each bird was challenged with 106 CFU/chick of SE by oral gavage $(0.5 \mathrm{~mL})$.

\section{Immunohistochemistry}

Immunohistochemical reactions were performed according to Hsu et al. (1981) for tissue cuts embedded in paraffin.

All collected intestinal fragments were fixed in 10\% Bouin solution for 24 hours and embedded in paraffin. Sections were cut to a thickness of $3 \mathrm{~mm}$, cleared in xylene, rehydrated in graded ethanol series, and rinsed in distilled water. Endogenous peroxidases were neutralized with $3 \%$ hydrogen peroxide for 15 minutes, followed by rinsing for 5 minutes in distilled water. Antigen was retrieved by incubating slides in EDTA solution (ethylenediaminetetraacetic acid disodium salt), $\mathrm{pH} 8.0$, in a steamer at $96^{\circ} \mathrm{C}$ for 30 minutes.

Nonspecific immunoglobulin was blocked by incubating the slides for 60 minutes with $5 \%$ bovine serum albumin (BSA) before application of the primary antibody. Slides were incubated for 18 hours at $40 \mathrm{C}$ with a monoclonal anti-chicken CD4+ T-lymphocytes (UNLB, Southern Biotechnology Associates, Inc.) at 1:25 dilution and monoclonal anti-chicken CD8+/144B Tlymphocytes (Dakocytomation CD8, T-cell BGM 7103) at 1:100 dilution. A streptavidin-immunoperoxidase staining procedure (LSAB) was used for immunolabeling CD8+ positive cells. CSA procedure was used for immunolabeling CD4+ positive cells. The immunoreactions were observed with 3' 3diaminobenzidine substrate (DAB). Sections were counterstained with Mayer's hematoxylin.

The negative controls were tested with mouse immunoglobulins (Ig) (for the monoclonal antibody); with one slide with only secondary antibody and one with only streptoavidine and peroxidase. All tested slides were negative.

After the immunohistochemical reactions, marked cells were counted under an optical microscope (JENAMED 2 - Carlzeiss Jena) with 40x magnification. CD4+ and CD8+ lymphocytes were counted in ten random fields in each slide of the epithelium and of the lamina propria of each intestinal segment (duodenum, jejunum, and cecum).

\section{Statistical analysis}

Data were submitted to analysis of variance using a completely randomized experimental design (Zar, 1996) in a factorial arrangement. Treatments consisted of combinations of the following factors: 1) oral treatment - CM, L. acidophillus, L reuteri, or L. salivarius; 2) challenge - treated and challenged birds, treated birds, challenged birds (positive control), and birds neither treated nor challenged (negative control); 3 ) bird age - birds of two, four, eight, and 12 days of age. There were five replicates per treatment, which were compared as to their effects on CD4+ and CD8+ lymphocyte counts at a $5 \%$ level of significance.

\section{RESULTS}

Positive reactions for CD4+ lymphocytes in the lamina propria of the ceca of two-day-old chicks treated with $L$. reuteri are illustrated in Figure 1. Figure 2 depicts the positive reaction for CD8+ lymphocytes in the duodenum of eight-day-old birds treated with $L$. salivarius.

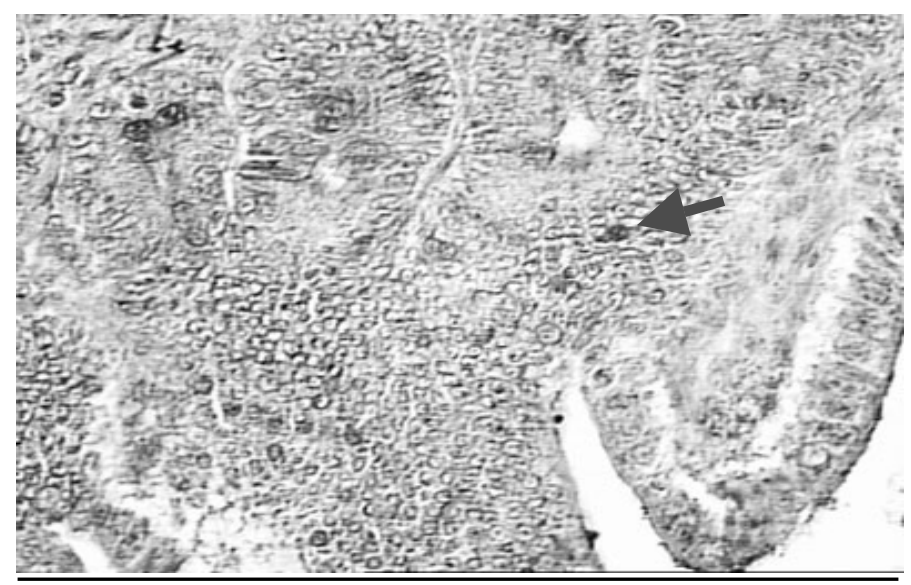

Figure 1 - Positive reaction of CD4+ lymphocytes (arrow) in the lamina propria of the cecum of 2-day-old chicks treated with Lactobacillus reuteri (kit CSA, DAB for 30 seconds, Mayer's hematoxylin counterstaining, 40x).

Table 1 presents the results of mean CD4+ lymphocyte counts obtained with treatment with 
Noujaim JC, Andreatti Filho RL, Lima ET de, Okamoto AS

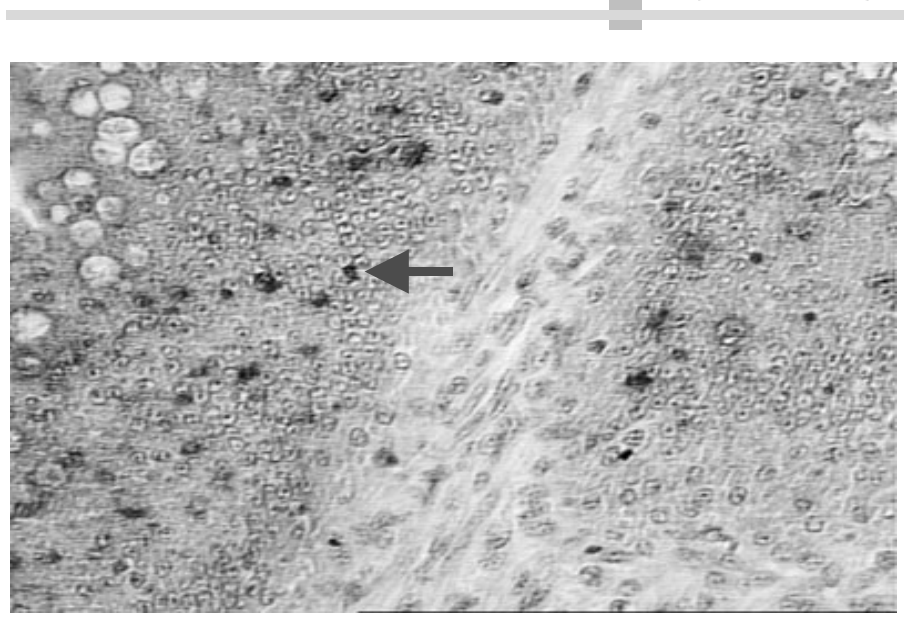

Figure 2 - Positive reaction of CD8+ lymphocytes (arrow) in the lamina propria of the duodenum of 8-day-old chicks treated with Lactobacillus salivarius (kit LSAB, DAB for five minutes, Mayer's hematoxylin counterstaining, 40x).

Lactobacillus spp. or CM, and challenge or not with SE according to bird age. The data show that at the first three ages (two, four, and eight days of age), no differences were observed $(p<0.05)$ between the negative control and the group treated with $\mathrm{L}$. salivarius and challenged with $S E$. Furthermore, it is observed that CD4+ lymphocyte mean counts of negative controls and birds treated with $L$. salivarius and $L$. acidophillus and challenged with SE were lower than those obtained in the other groups. However, at the fourth age (12 days of age), there was a difference $(p<0.05)$ in CD4+ lymphocyte counts between the negative control and the groups treated with $L$. reuteri, L salivarius, L. acidophilus, or cecal microbiota and challenged with SE. There was no effect of bird age $(p<0.05)$ on CD4+ T cell counts.

\begin{tabular}{|c|c|c|c|c|}
\hline \multirow[b]{2}{*}{ Treatment } & \multicolumn{4}{|c|}{ Age (days) } \\
\hline & 2 & 4 & 8 & 12 \\
\hline Negative control & $3.4 \mathrm{BCa}$ & 3.1Ba & $2.4 \mathrm{Aa}$ & $3.9 \mathrm{Ca}$ \\
\hline$S E$ & $8.0 \mathrm{BC}$ & $6.9 \mathrm{ABb}$ & $6.2 \mathrm{Ab}$ & $6.6 \mathrm{Ab}$ \\
\hline L. reuteri & $8.1 \mathrm{BC}$ & $7.8 \mathrm{BC}$ & $6.4 \mathrm{Ab}$ & $6.8 \mathrm{Ab}$ \\
\hline L. salivarius & $6.7 \mathrm{ABb}$ & $7.4 \mathrm{BC}$ & $6.0 \mathrm{Ab}$ & 7.0ABb \\
\hline L. acidophilus & $6.7 \mathrm{ABb}$ & $7.4 \mathrm{BC}$ & 5.9Ab & $6.9 \mathrm{ABb}$ \\
\hline $\mathrm{CM}$ & $8.1 \mathrm{BC}$ & $7.8 \mathrm{BC}$ & $6.4 \mathrm{Ab}$ & $6.8 \mathrm{Ab}$ \\
\hline L. reuteri + SE & $8.9 B C$ & $6.5 \mathrm{Ab}$ & $6.1 \mathrm{Ab}$ & $6.1 \mathrm{Ab}$ \\
\hline L. salivarius + SE & 3.6Aa & 3.3Aa & 3.4Aa & $6.1 \mathrm{Bb}$ \\
\hline L. acidophillus + SE & $3.6 \mathrm{Aa}$ & 3.3Aa & $3.4 \mathrm{Aa}$ & $3.1 \mathrm{Aa}$ \\
\hline$C M+S E$ & $8.9 B C$ & $6.5 \mathrm{Ab}$ & $6.1 \mathrm{Ab}$ & $6.1 \mathrm{Ab}$ \\
\hline
\end{tabular}

Upper-case letters compare mean ages for each treatment (Row). Lower-case letters compare means of treatment for each age (Column). Means followed by at least one same letter are not significantly different $(p<0.05)$.
Detection of CD4+ and CD8+ Lymphocytes in the Intestine of Broiler Chicks Treated with Lactobacillus spp. and Challenged with Salmonella enterica serovar Enteritidis

Average CD4+ lymphocyte counts obtained after birds were orally treated Lactobacillus spp. or CM and challenged or not with SE in relation to the different intestinal segments of chicks are shown in Table 2. There was a difference $(p<0.05)$ between average CD4+ lymphocyte counts between the negative controls and birds treated with Lactobacillus spp or CM, challenged or not with $S E$, in the duodenum and jejunum of chicks. In addition to the significant difference between the average CD4+ lymphocyte counts, their values are higher in the treated groups as compared to the negative control. Moreover, in relation to the ceca, there was no difference $(p<0.05)$ between the negative control and the treatments with L. salivarius and $L$. acidophillus and challenged with SE.

\begin{tabular}{|c|c|c|c|}
\hline \multirow[b]{2}{*}{ Treatment } & \multicolumn{3}{|c|}{ Segment } \\
\hline & Duodenum & Jejunum & Ceca \\
\hline Negative control & $3.5 \mathrm{Aa}$ & $3.1 \mathrm{Aa}$ & $3.0 \mathrm{Aa}$ \\
\hline$S E$ & $7.7 \mathrm{BC}$ & 6.8Ade & $6.3 \mathrm{Abc}$ \\
\hline L. reuteri & 7.4ABC & $7.9 \mathrm{Bf}$ & $6.5 A C$ \\
\hline L. salivarius & $7.9 \mathrm{BC}$ & 6.6Acd & $5.8 \mathrm{Ab}$ \\
\hline L. acidophilus & $7.8 \mathrm{BC}$ & $6.1 \mathrm{Ac}$ & $5.8 \mathrm{Ab}$ \\
\hline CM & 7. $4 \mathrm{ABC}$ & $7.9 \mathrm{Bf}$ & $6.5 A c$ \\
\hline L. reuteri + SE & 7.0AC & 7.1Ae & $6.5 \mathrm{Ac}$ \\
\hline L. salivarius + SE & $4.5 \mathrm{Bb}$ & $4.6 \mathrm{Bb}$ & 3.3Aa \\
\hline L. acidophillus + SE & $4.5 \mathrm{Bb}$ & $4.6 \mathrm{Bb}$ & 3.3Aa \\
\hline$C M+S E$ & 7.0AC & 7.1Ae & $6.5 \mathrm{Ac}$ \\
\hline
\end{tabular}

Upper-case letters compare mean ages for each treatment (Row). Lower-case letters compare means of treatment for each age (Column). Means followed by at least one same letter are not significantly different $(p<0.05)$.

Average CD8+ lymphocyte counts in the intestine of chicks, obtained after treatments with Lactobacillus spp. or CM and challenge or not with SE related to different chick ages are shown in Table 3. Analyzing CD8+ lymphocyte counts, a difference was found $(p<0.05)$ between the negative control and the other groups, independent of oral treatment and/or challenge. The chicks treated with CM and challenged with SE presented the highest CD8+ lymphocyte counts in eight- and 12-day-old chicks.

Table 4 shows average CD8+ lymphocyte counts in the duodenum, jejunum and ceca of chicks treated with Lactobacillus spp. or CM and challenged or not with $S E$. The negative control was significantly different 
Noujaim JC, Andreatti Filho RL, Lima ET de, Okamoto AS
Detection of CD4+ and CD8+ Lymphocytes in the Intestine of Broiler Chicks Treated with Lactobacillus spp. and Challenged with Salmonella enterica serovar Enteritidis $(p<0.05)$ from the other groups, again with higher lymphocyte counts in the treated groups as compared to the negative control. Comparing mean cell counts among the different intestinal segments of the same treatment, no difference was observed $(p<0.05)$ between the negative control and the treatments with $L$. salivarius and L. acidophillus challenged with SE. The chicks treated with CM and challenged with SE and only challenged with SE presented the highest CD8+ lymphocyte counts in the duodenum, jejunum, and ceca.

\begin{tabular}{lcccc}
\hline Table 3 - Mean quantities of CD8+ lymphocytes marked with \\
specific antibody by immunohistochemistry and counted in ten \\
random fields in each tissue section of the duodenum, jejunum, \\
and ceca of chicks orally treated with Lactobacillus spp. or cecal \\
microflora (CM) and challenged or not with Salmonella enterica \\
serovar Enteritidis (SE) according to bird age (C.V.=26.7\%). \\
\hline \multicolumn{5}{c}{ Age (days) } \\
Treatment & $\mathbf{2}$ & $\mathbf{4}$ & $\mathbf{8}$ & $\mathbf{1 2}$ \\
\hline Negative control & $9.5 \mathrm{Ba}$ & $7.0 \mathrm{Aa}$ & $6.8 \mathrm{Aa}$ & $10.6 \mathrm{Ba}$ \\
SE & $25.2 \mathrm{Bg}$ & $25.4 \mathrm{Be}$ & $24.0 \mathrm{Bf}$ & $18.0 \mathrm{Acd}$ \\
L. reuteri & $17.1 \mathrm{Ade}$ & $13.9 \mathrm{Ab}$ & $15.3 \mathrm{Ab}$ & $22.0 \mathrm{Be}$ \\
L. salivarius & $16.8 \mathrm{Ad}$ & $20.4 \mathrm{Bd}$ & $23.8 \mathrm{Cf}$ & $15.6 \mathrm{Ab}$ \\
L. acidophilus & $15.8 \mathrm{Acd}$ & $18.0 \mathrm{ABC}$ & $19.3 \mathrm{Bcd}$ & $19.0 \mathrm{Bd}$ \\
CM & $21.7 \mathrm{Bf}$ & $19.8 \mathrm{ABd}$ & $22.1 \mathrm{Be}$ & $17.4 \mathrm{Ac}$ \\
\hline L. reuteri + SE & $18.7 \mathrm{ABe}$ & $16.9 \mathrm{Ac}$ & $21.0 \mathrm{Bde}$ & $21.4 \mathrm{Be}$ \\
L. salivarius + SE & $14.6 \mathrm{Abc}$ & $14.9 \mathrm{ABbc}$ & $17.9 \mathrm{Bc}$ & $16.2 \mathrm{Bbc}$ \\
L. acidophilus + SE & $12.4 \mathrm{Ab}$ & $14.1 \mathrm{ABbc}$ & $15.9 \mathrm{Bb}$ & $15.1 \mathrm{Bb}$ \\
CM + SE & $23.1 \mathrm{Afg}$ & $24.9 \mathrm{ABe}$ & $27.3 \mathrm{Cg}$ & $25.5 \mathrm{BCf}$ \\
\hline
\end{tabular}

Upper-case letters compare mean ages for each treatment (Row). Lower-case letters compare means of treatment for each age (Column). Means followed by at least one same letter are not significantly different $(p<0.05)$.

\begin{tabular}{llll}
\hline Table $\mathbf{4}$ - Mean quantities of CD8+ lymphocytes marked with \\
specific antibody by immunohistochemistry and counted in ten \\
random fields in each tissue section of chicks orally treated with \\
Lactobacillus spp. or cecal microflora (CM) and challenged or \\
not with Salmonella enterica serovar Enteritidis (SE) according \\
to intestinal segment (C.V.=26.7\%). \\
\hline \multicolumn{4}{c}{ Segment } \\
\cline { 2 - 4 } Treatment & Duodenum & Jejunum & Ceca \\
\hline Negative control & $8.8 \mathrm{Aa}$ & $8.4 \mathrm{Aa}$ & $8.2 \mathrm{Aa}$ \\
SE & $25.3 \mathrm{Cf}$ & $23.7 \mathrm{Bf}$ & $20.3 \mathrm{Ade}$ \\
L. reuteri & $18.5 \mathrm{Bcd}$ & $17.7 \mathrm{BC}$ & $14.9 \mathrm{Abc}$ \\
L. salivarius & $20.3 \mathrm{Ade}$ & $17.7 \mathrm{Ac}$ & $19.5 \mathrm{Ad}$ \\
L. acidophilus & $20.6 \mathrm{Bde}$ & $18.9 \mathrm{Bd}$ & $14.3 \mathrm{Ab}$ \\
CM & $20.6 \mathrm{ABde}$ & $22.0 \mathrm{Be}$ & $18.1 \mathrm{Ad}$ \\
L. reuteri + SE & $21.4 \mathrm{Be}$ & $20.7 \mathrm{Bde}$ & $16.3 \mathrm{Ac}$ \\
L. salivarius + SE & $16.6 \mathrm{Abc}$ & $15.1 \mathrm{Ab}$ & $16.0 \mathrm{Ac}$ \\
L. acidophillus + SE & $14.9 \mathrm{Ab}$ & $14.1 \mathrm{Ab}$ & $14.1 \mathrm{Ab}$ \\
CM + SE & $27.5 \mathrm{Bf}$ & $25.8 \mathrm{Bf}$ & $22.4 \mathrm{Ae}$ \\
\hline
\end{tabular}

Upper-case letters compare mean ages for each treatment (Row). Lower-case letters compare means of treatment for each age (Column). Means followed by at least one same letter are not significantly different $(p<0.05)$.

\section{DISCUSSION}

In the present study, samples of Lactobacillus reuteri, L. salivarius, L. acidophilus and CM isolated from breeders supplied by oral gavage to chicks challenged or not with SE demonstrated capacity to stimulate the immune system, in the form of leukocytic infiltrate of CD4+ and CD8+ lymphocytes in the intestinal epithelium and lamina propria of chicks as detected by immunohistochemistry. Thus, the results obtained in the present study agree with Lillehoj \& Chung (1992), who asserted that the development and composition of intestinal T-cells are influenced by the exposure to environmental antigens, suggesting that the presence of such lymphocytes in the intestinal epithelium is due to intraluminal antigenic stimulus.

A similar result was obtained by Vervelde et al. (1998), who identified, also by immunohistochemistry, a high quantity of leukocytic infiltrate of CD3+ lymphocytes, and particularly of CD4+ and CD8+ cells, in the epithelium and in the lamina propria of chick's intestine seven days after the treatment with a mixture of recombinant antigen of Eimeria and choleric toxin. Our results are also consistent with the assertion of Elwood et al. (1997) that in humans, swine, and rodents, T-cells are deposited as much in the intestinal epithelium as in the intestinal lamina propria. In addition to identifying these same structures, another marked similarity between the study by Vervelde et al. (1998) and the present study was that, in both, CD8+ lymphocyte counts were higher than those of CD4+.

McSorley et al. (2000) demonstrated that a significant fraction of Salmonella-specific CD4+ T-cells respond to the flagellar filament protein, $\mathrm{FliC}$, and that this antigen is capable of protecting against lethal Salmonella infection. Choi et al. (1999) described the alterations in T-cell subpopulations, including CD4+, CD8+, TCR1, and TCR2 lymphocytes, as well as the transcription of IFN- $\gamma$, and TGF-B4 mRNA in the intestine of chicks after oral inoculation with $E$. acervulina. According to Songserm et al. (2002), the influx of Tcells into the intestinal epithelium is related to the presence of bacteria in the intestinal lumen. Those authors suggest that cell-mediated intestinal immunity is directly related with the elimination of enteropathogens, such as Pasteurella multocida and Eimeira, from the intestine of chicks. Therefore, the results obtained by those researchers are consistent with the present experiment, where an increase in CD4+ and CD8+ lymphocytes population was shown four days after the beginning of treatments. 
Noujaim JC, Andreatti Filho RL, Lima ET de, Okamoto AS
Detection of CD4+ and CD8+ Lymphocytes in the Intestine of Broiler Chicks Treated with Lactobacillus spp. and Challenged with Salmonella enterica serovar Enteritidis
Yet, according to Songserm et al. (2002), in addition to bacterial stimulation, the increase in chick age is responsible for the influx of lymphocytes into the intestinal epithelium of chicks. A study performed by Lillehoj \& Chung (1992) with broiler chickens verified that the percentage of CD3+ lymphocytes increased $24.4 \%$ by one week of age, $42 \%$ by the second week, and $62.6 \%$ by the fourth week. In relation to the variation in the number of CD4+ lymphocytes with chick age, the percentages obtained in the present study also agree with the findings of Lillehoj \& Chung (1992), who observed a decrease in CD4+ lymphocyte counts as chicks aged. Still in relation to CD8+ lymphocyte counts, the numbers obtained by Lillehoj \& Chung (1992) are different from those of present study as they showed a gradual and discrete increase in the number of these cells as the birds aged.

Thus, it can be concluded that the oral treatment with $L$. reuteri, $L$. salivarius, $L$. acidophilus, or CM of chicks challenged or not with SE elicit an immune response in the form of leukocytic infiltrate by CD8+ lymphocytes, followed by CD4+, in the intestine in the period from two to 12 days of age. CD8+ lymphocyte counts were significantly higher in the intestine of chicks treated with $\mathrm{CM}$ and challenged with SE. The duodenum, followed by the jejunum, were the segments in which the immune response by $T, C D 4+$, and CD8+ cells was stimulated with the strongest intensity.

\section{REFERENCES}

Andreatti Filho RL, Sampaio HM, Barros MR, Gratao PR, Cataneo A. Use of cecal microflora cultured under aerobic or anaerobic conditions in the control of experimental infection of chicks with Salmonella Enteritidis. Veterinary Microbiology 2003; 92:237-244.

Centers for Disease Control and Prevention. Salmonella Surveillance: annual summary. Atlanta (GA): US Department of Health and Human Services; 2005.

Day CA. Competitive exclusion in poultry: a review. Worcessershire: Life-are; 1992. Production, 18.

Elwood CM, Hamblin AS, Batt RM. Quantitative and qualitative immunohistochemistry of T cell subsets and MHC Class II expression in the canine small intestine. Veterinary Immunology Immunopathology 1997; 58:195-207.

Gast RK, Beard CW. Production of Salmonella enteritidiscontaminated eggs by experimentally infected hens. Avian Diseases 1990; 34:438-446.

Hsu HM, Raine L, Fanger $\mathrm{H}$. Use of avidine-biotine-peroxidase complex $(A B C)$ in immunoperoxidase techniques: A comparison between $A B C$ and unlabeled antibody (PAP) procedure. Journal of Histochemistry Cytochemistry 1981; 29:577-580.

Joerger RD. Alternatives to antibiotics: bacteriocins, antimicrobial peptides and bacteriophages. Poultry Science 2003; 82:640-647.

Kandler O, Weiss R. Section 14, Nonsporing Gram-positive Roods. In: Sneth PHA, Maine NS, Sharpe ME, Holt JG, editors. Proceeding of the Bergey's Manual of Systematic Bacteriology. Baltimore; 1986. p.1208-1234.

Lillehoj HS, Chung KS. Postnatal development of T-lymphocyte subpopulations in intestinal intraepithelium and lamina propria in chickens. Veterinary Immunology Immunopathology 1992; 31:347360.

Lilly DM, Stillwell RH. Probiotics: growth promoting factors produced by microorganisms. Science 1965; 147:747-748.

McSorley SJ, Cookson BT, Jenkins MK. Characterization of CD4+ T cell responses during natural infection with Salmonella typhimurium. The Journal of Immunology 2000; 164:986-993.

Molbak K, Neimann J. Risk factors for sporadic infection with Salmonella enteritidis, Denmark, 1997-1999. American Journal of Epidemiology 2002; 156:654-661.

Muir WI, Bryden WL, Husband AJ. Immunity vaccination and the avian intestinal tract. Development Compared Immunology 2000; 24:325-342.

Nagajara KV, Pomeroy BS, Willians JE. Paratyphoid Infections. In: Calnek BW, editor. Diseases of poultry. 9th ed. Ames: State University Press; 1991. p.99-130.

Pelto L, Isolauri E, Lilius EM, Nuutila J, Salminen S. Probiotic bacteria down-regulate the milk-induced inflamatory response in milkhypersensitive subjects but have na immunostimulatory effect in healthy subjects. Clinical Experimental Allergy 1998; 28(12):14741479.

Pulverer G, Ko HL, Roszkowski W, Beuth J, Yassin A, Jeljaszewicz J. Digestive tract microflora liberates low molecular weight peptides with immunotriggering activity. International Journal of Medical Microbiology 1990; 272:318-327.

Ramesh BK, Satynarayana ML, Gowda RNS, Vijayasarathi SK, Suguna RAO. Effect of Lactobacillus acidophilus on gut $\mathrm{pH}$ and viable bacterial count in experimental fowl typhoid in broilers. Indian Veterinary Journal 2000; 77:544-546.

Schiffrin EJ, Brassart D, Seruin AL. Immune Modulation of blood leukocytes in humans by lactic acid bacteria: criteria from strain selection. American Journal of Clinical Nutrition 1997; 66:515520.

Simon O, Jadamus A, Vahjen W. Probiotic feed additives effectiveness and expected modes of action. Journal of Animal Feed Science 2001; 10:51-67.

Songserm TH, Engel B, Roozelaar DJ, Kok GL, Pijpers A, Pol JMA, Huurne AAHM. Cellular immune response in the small intestine of 
Noujaim JC, Andreatti Filho RL, Lima ET de, Okamoto AS

two broiler chicken lines orally inoculated with malabsorpstion syndrome homogenates. Veterinary Immunology Immunopathology 2002; 85:51-62.

Sulakvelidze A, Alavidze Z, Morris Jr. JG. Bacteriophage therapy. Antimicrobe Agents and Chemotherapy 2001; 45:649-659.

VanImmerseel F, Cauwerts K, Devriese LA, Haesenbrouck F, Ducatelle R. Feed additives to control Salmonella in poultry. World's Poultry Science Journal 2002; 58:501-513.

Vervelde L, Janse EM, Vermeulen AN, Jeurissen SHM. Induction of local and systemic immune response using cholera toxin as vehicle to deliver antigen in the lamina propria of the chicken intestine. Veterinary Immunology Immunopathology 1998; 62:261-272.

Weinack OM, Snoeyenbos GH, Smyser CF, Soerjadi AS. Reciprocal competitive exclusion of Salmonella and Escherichia coli by native intestinal microflora of the chicken and turkey. Avian Diseases 1982; 26:585-95.

Zar JH. Biostatistical Analysis. New Jersey: Prentice Hall; 1996. p.718. 


\section{Conferência FACTA 2010}

\section{5 a 27 de Maio de 2010 Santos - SP - Brasil}

Em breve maiores informações:www.facta.org.br 